\title{
How to get published in JoMaC
}

\author{
Thomas W. Guenther
}

(C) Springer-Verlag Berlin Heidelberg 2014

Very often the editors of the Journal of Management Control are asked what scholars can do to successfully publish their papers in our journal. In general, the answer is twofold.

On the one hand, as for all academic journals, the same requirements for a good paper also hold for papers submitted to the Journal of Management Control: an innovative idea contributing to current research, a solid and high quality application of research methods, an understandable and easy to read writing style and a good organization of the paper (informative introduction, sufficient reference to current research, explanation of the research gap and of the contribution of the paper, solid presentation of the methods and their adequacy, understandable presentation and discussion of results, testing for the robustness of the results, explanation of limitations of the chosen approach, conclusions and contributions to current research). This is neither surprising, nor unique and is consistent with the nature of all academic journals with the ambition to make a contribution to the research in their specific field.

On the other hand, looking back on more than two decades of experience with our specialized journal and now some years as an English language journal, we would like to add some additional aspects which might be specific for our field of research in management control as well as for the Journal of Management Control especially:

Looking for new methods Scholars often criticize the difficulties they perceive when attempting to publish papers which use non-mainstream methods which are not so well explored and well experienced as regression or structural equation modeling. I personally remember a statement of a highly reputable managing editor of a very influential accounting journal, saying that a specific (new) method will never be published in his

T. W. Guenther $(\varangle)$

Journal of Management Control, Technische Universität Dresden, 01062 Dresden, Germany

e-mail: thomas.guenther@tu-dresden.de 
journal. How can we say this? Each method has advantages and disadvantages and can be used for different aspects of our research. JoMaC as a rather young journal in the international accounting fields is open to explore new methods under the condition that they are solidly performed and adequately used.

Looking for new data Discussing with colleagues and with Ph.D. students in accounting one often gets the impression that archival data is the ultimate solution and data source for almost any question because it is regarded to be objective and because sample sizes are in general large. We stretch proxies widely without asking where data come from or what we really want to measure. And if we do not have data on something it is not regarded as an issue, not measurable or - even worse - not relevant. We also face prejudices against subjective, hand-collected and/or survey data because it is regarded as not being objective. However, we never look at how archival data is generated, how firms differently report financial accounting data, what you really find in published annual reports in relation to what we find in data bases and so on. Thus, we welcome different data sources. We inspire scholars to utilize unique data sources such as thoroughly and validly hand-collected data, combining different data sources like in dyadic research or combing survey and archival data.

Replicating studies/conducting meta-analyses In our field of research, management control and management accounting, we often find only one or a limited number of studies on the specific interaction between two variables. Most journals always ask how can we expand existing literature? What is new? Pure replicating studies are mostly not acknowledged and often not accepted. Often reviewers remark that this result is already known to the community and therefore obvious. Nevertheless, there is some need for replication and management control should look on other fields such as medicine or psychology with a much larger body of studies and a strong history of literature reviews and meta-analyses. In these fields of research getting one significant results on an interaction between two variables is not sufficient. In medicine we look for evidence based medicine. In management control meta-analyses often cannot be run as the sample sizes of primary studies are below six, the absolute minimum to get aggregated population effects. But, what do we know about French service firms when we have results for the US manufacturing sector which are ten years old? Is the context the same, doesn't it change over time? I personally still see enough room for replication studies which not purely replicate other studies, but expand them to explain the impact of different industries, strategies, firm sizes etc. There seems to be a need also for evidence-based management.

Helping papers to develop We want to inspire our reviewers neither to kill nor to promote papers for which they are invited to review. This message is not easy to communicate. Nevertheless, it should be our duty as reviewers to give fruitful comments to authors and not to give them grades or to show them that they are "bad" scholars. I am convinced that each paper can be developed and that each author wants to do her or his best. Thus, we try to give detailed comments to authors, making specific suggestions, delivering helpful references or sources that allow authors to improve their paper for the next round of review. Knowing that some journals have too many submissions and thus have to be quite strict in reviewing, we try to offer a second round to revise and resubmit the paper even if it is risky and even if the paper 
will not finally make it. But, authors get a chance to learn from the review process and to improve their paper.

Listen to reviewers, be humble, ask for guidance Whereas the above section was referring to the job of the reviewers, also the authors have to do a professional job. I realize that sometimes authors try to ignore, comments of reviewers and try to get around them too easily which sometimes frustrates good-hearted reviewers. Authors should carefully listen to comments and recommendations of reviewers, try to be humble and to think how a specific remark can improve the paper. We also face the fact that authors are inclined to withdraw their paper if the path ahead seems to be too difficult. But what is the alternative? Start from scrath on at another journal with a similar outcome? I cordially invite both authors and reviewers to ask for guidance if they do not know which path to take and what to do.

When the going get's tough, the tough get's going This American proverb sometimes helps if a review process takes too long. I remember one author in the last months who declared his wishes to withdraw, which I did not understand because he was so close to publication. We finally rescued him from "self-destruction" after gently handling his case. I realize that after Ph.D. students receive their degree they are often no longer interested in publication which I personally do not understand. "It is not research until it is published." This wording of one of our American guest professors which is certainly driven by her life experience should be kept in our minds and we should train our young scholars in this mind set. "Publication is nights and weekends" is the experience of life long learning process of another well experienced colleague in management science which may shock younger generations, but seems rather realistic when you intend to follow an academic career.

Also in this issue it is my pleasure to present some papers which have obviously regarded the above advice because they have finally made it. This issue also reflects the above mentioned as the papers present different data sources, different methodologies, and different focuses in the wide field of management control. Nevertheless, four papers with authors from four different countries underline the intended international reach of our journal.

Alexander Brüggen and Jens Oliver Zehnder explore the cost stickiness of SG\&A costs using regression modeling and ask whether cost stickiness is influenced by equity-based executive compensation.

The relationship of management accounting information and financial performance is the focus of the study of Erkki Kalervo Laitinen who examines a setting of Finnish top managers. Applying a partial least squares (PLS) method he analyzes survey data from 215 Finnish CEOs.

Orhan Akisik and Graham Gal analyze the association between CSR reviews using data from the Global Reporting Initatives (GRI) and financial performance for a setting of North American firms for the period between 2006 and 2012. They combine two different data sources, GRI and archival data from Compustat, using regression modelling.

Finally, Lauri Lepistö conducts an empirical analysis which draws upon the critical discourse analysis that is part of the qualitative research tradition. He applies the method to articles on ERP system adoptions published in the customer relationship magazine of a notable accounting software company covering the years 2002-2012, a 
quite unique data source seldomly used in management control research, which might inspire the use of other unique data sources.

Dresden, December 2014

Prof. Dr. Thomas Guenther

Managing editor 